\title{
Covid-19 Cases
}

Oliveiros $\mathrm{B}^{1},{ }^{2},{ }^{3 *}$, Caramelo $\mathrm{L}^{4}$, Ferreira $\mathrm{NC}^{1},{ }^{3}$, Caramelo $\mathrm{F}^{1},{ }^{2,}{ }^{3}$

'Laboratory of Biostatistics and Medical Informatics, Faculty of Medicine, University of Coimbra, Portugal

${ }^{2} i c b r$ - Coimbra Institute for Clinical and Biomedical Research, Faculty of Medicine, University of Coimbra, Portugal

${ }^{3} \mathrm{CIBIT}$ - Coimbra Institute for Biomedical Imaging and Translational Research, University of Coimbra, Portugal

${ }^{4}$ Centre for the Research and Technology of Agro-Environmental and Biological Sciences, CITAB, University of Trás-os-

Montes and Alto Douro, Vila Real, Portugal

*Corresponding author: Bárbara Oliveiros, 1Laboratory of Biostatistics and Medical Informatics, Faculty of Medicine, University of Coimbra, Portugal

\section{Abstract}

Objectives: To estimate influence of meteorological variables, notabl temperature and relative humidity, on the spreading of the SARS- CoV-2 virus. Adjusted odds ratio were used to quantify the influence of temperature and relative humidity on the doubling time of the spread.

Setting: The data regarding the China COVID-19 outbreak used in this work were retrieved from the Johns Hopkins University COVID-19 dashboard. The meteorological data was obtained using the Meteostat Application Programming Interface.

Participants: No patients were directly included in this study since data were collected from internet sites. Primary and secondary outcome measures: the primary outcome measured was the number of confirmed infected and the secondary outcomes were temperature, relative humidity, wind speed and precipitation of the China provinces in the period of analysis.

Results: Results indicate that the doubling time correlates positively with temperature and inversely with relative humidity. We found that, at maximum, an average temperature increase of $1{ }^{\circ} \mathrm{C}$ augments the doubling time in 0.090 days and an increase of $1 \%$ of relative humidity leads to a decrease of 0.072 days in the doubling time.

Conclusion: The results obtained suggest that decrease in the rate of progression of COVID-19 with the arrival of spring and summer in the north hemisphere since a $20^{\circ} \mathrm{C}$ increase is expected to delay the doubling time in 1.8 days in average. Temperature and relative humidity only explain $18 \%$ of the variation in disease doubling time; the remaining $82 \%$ may be related to containment measures, general health policies, population density, transportation or cultural aspects.

Trial Registration: Not applicable.

\section{Strengths of this Study}

It is established a quantitative relationship between the rate of progression of COVID-19 and temperature and relative humidity

The model is easily generalized to other situations

Limitations of this Study

Only the exponential portion of the outbreak is used to determine the relationship between the variables

There is uncertainty linked to the meteorological variables concerningtheir regional representatively as only one value is used to characterized a whole region

Keywords: COVID-19; doubling time; temperature; relative humidity; precipitation; wind speed; mortality 
Introduction

The outbreak of pneumonia cases in Wuhan, China during last December led to great efforts to prevent a global epidemic. The alert from China CDC was rapidly transmitted to the World Health Organization [1-3] excluding possible causes such as influenza, adenovirus, severe acute respiratory syndrome (SARS-CoV) and Middle-East respiratory syndrome (MERS-CoV)[1,3-4]. The novel coronavirus, named SARS-CoV-2, and its genomic characterization was performed a few days after, permitting to devise a robust test method [1-5]. Although the genomic characterization revealed some relations both to SARS- CoV and MERS-CoV, [4-6] the new virus was found to be much more aggressive than those other coronaviruses or the seasonal one [4-6]. When human-to-human transmission was proved, on the 20th of January, the onset of the disease (COVID-19) has changed [2,7-10]. According to the China CDC11, the case fatality rate (CFR) was $0.2 \%$ at the end of January 2020 and $14.4 \%$ of the confirmed cases were considered severe or even critical. In the last week of February, 79441 cases were confirmed worldwide ( $97 \%$ in Mainland China) and the number of deaths was 2620 (95\% in Mainland China12,13). The epidemiological curves of COVID-19 in China showed the progression of illness in the outbreak over time from December 8, 2019 up to February 11, 2020,11 when there was a total of 72314 confirmed cases as the geo-temporal spread of COVID-19 [11].

At that time, the majority of confirmed cases occurred in the norther hemisphere and until the last week of February 2020 no confirmed cases had been reported in South America or Africa, except for one case in Egypt $[12,13]$. In fact, the first confirmed case in Brazil was reported on February 26, while Algeria and Nigeria reported the first cases respectively on the 25th and 27th of February. The discussion about the COVID-19 epidemic spread in the northern hemisphere, while low temperatures and high relative humidity are present, and the unknown, although expectable positive impact of spring and summer in sustaining the epidemic, as its spread into the southern hemisphere was not as fast, has raised our question: how do meteorological variables, such as temperature and relative humidity, modulate COVID-19 duplication time?

Even though there is not yet strong evidence that meteorological conditions may have a role on COVID-19 outbreak or on human transmission, some studies have reported their role in guinea pigs' influenza transmission [14] and enveloped virus survival [15] in droplets. Some evidence of a faster spreading of diseases in high relative humidity levels has been reported [16], namely for the Legionella disease, although this infection is not caused by a virus. Some papers have been written since mid-February on this topic, [17-20] even though the relationship is not perfectly established and more research is required. We intend to add value to this discussion by evaluating the meteorological impact on COVID-19 duplication time.

\section{Material and Methods}

\section{Patient and Public Involvement}

\section{No patient involved}

\section{Methods}

The statistical model developed was implemented in two steps: firstly, an exponential model relating the accumulated number of confirmed cases and time was considered. Secondly, the rate of spread was used as dependent variable in a linear model that took as independent variables temperature, relative humidity, precipitation and wind speed. Only cases belonging to China were considered, as an attempt to control both cultural aspects and policies adopted to contain the virus. Therefore, data from the 31 provinces of Mainland China were gathered from the 23rd January up to the 1st of March, completing 39 days. These data were completed with meteorological variables, comprising temperature, relative humidity, precipitation and wind speed, collected for the same period, using the Meteostat Application Programming Interface (API) [21]. We searched for meteorological stations containing hourly measurements of these variables for the whole 39-day time period that were closest to the latitude and longitude coordinates that were available in the files that contained the confirmed cases time-series [13]. These geolocalization coordinates correspond to the geometrical centerpoint of each chinese province [22]. When meteorological data from a station was not possible to obtain around that position, another search point was chosen randomly from the set of closest nodes of an XY grid of nodes separated by 0.5 degrees in latitude and longitude and centered in the originally desired geolocation.

To compute the rate of spread a simple exponential model was assumed,

described by:

$=, \quad($ eq1 $)$

0

where 0 is the number of infected at instant zero, represents the rate of infection or the rate of spread and is the time. A more natural way of interpreting is by transforming it into the doubling time, , given by:

Citation: Oliveiros $\mathrm{B}^{1,2,3^{*}}$, Caramelo $\mathrm{L}^{4}$, Ferreira $\mathrm{NC}^{1,3}$, Caramelo $\mathrm{F}^{1,2,3}$. Role of Temperature and Humidity in the Modulation of the Doubling Time of Covid-19 Cases Op Acc J Bio Sci \& Res 7(1)-2021.

DOI: 10.46718/JBGSR.2021.08.000199 

(eq2)

The doubling time is the time needed to duplicate the number of infected subjects. Since the rate of progression changes over time and the exponential modelndoes not hold any longer, we considered mainly the initial days of the time series, selecting several periods of time composed each one of a predetermined number of consecutive days, but with different starting points. The starting point was assumed by randomly choosing the first day of the period. For each province several periods were sampled, allowing to obtain more than one value of the rate of progression (Figure 1).
Two different periods of time of 12 and 15 days were tested, along with 3 and 155 different starting points.

For each rate of progression, a corresponding value of each meteorological variable was computed, taking into account the same period of time. We opted to use the median of the meteorological variables (temperature, relative humidity, precipitation and wind speed) as it is more robust than the mean and tends to better represent the central tendency of the variable. Only models attaining more than 0.75 for the adjusted $\mathrm{R}$ square value were selected (Figure 1).

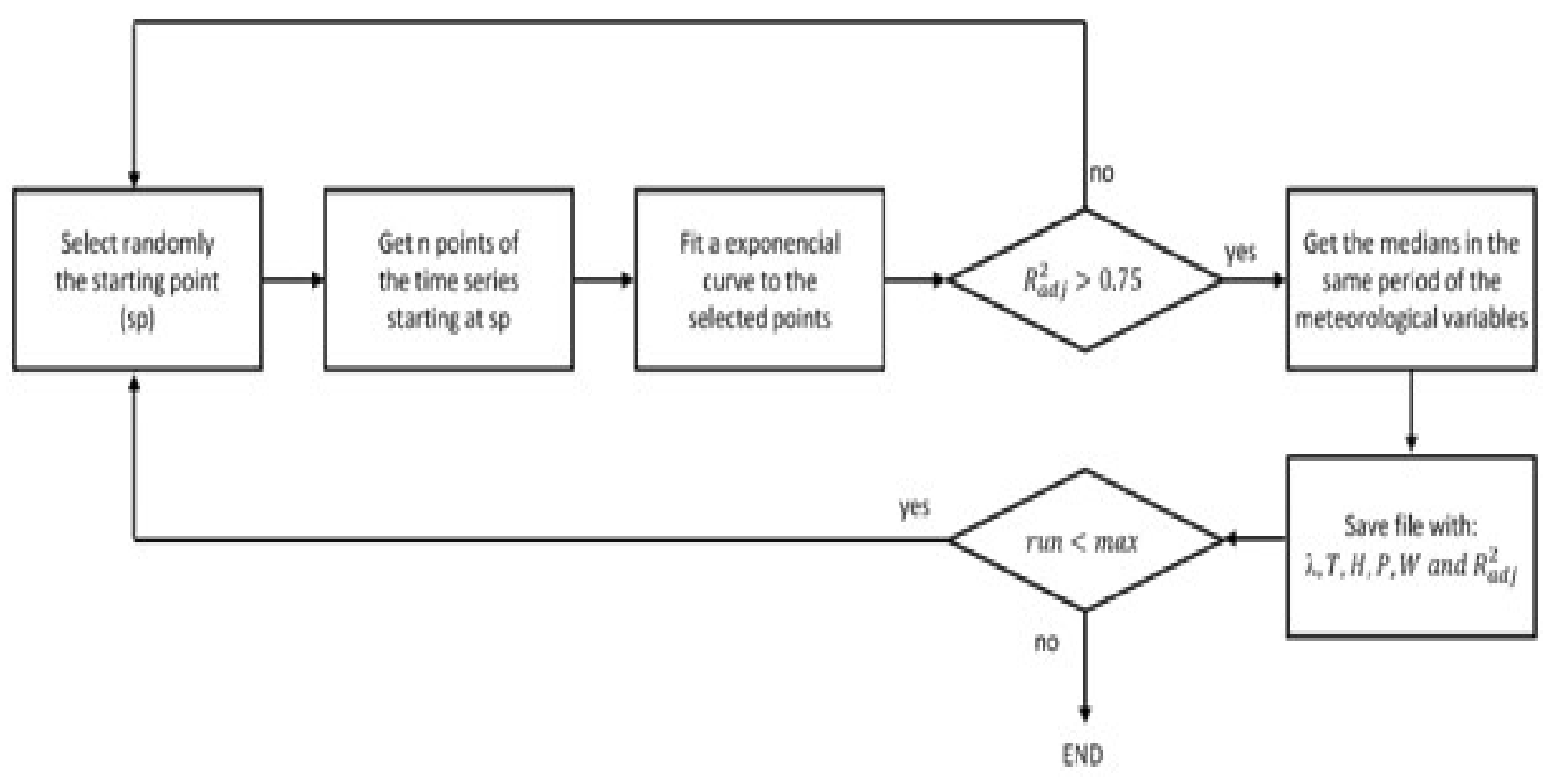

Figure 1: Flowchart of the routine in R language used to Compute the statistical models of Covid -19 Spreading of new cases.

The rate of progression was transformed into the doubling time, and recorded along with the median of temperature, relative humidity, precipitation and wind speed. These values were then used to fit a linear regression model aiming to assess how the meteorology is related to the doubling time. The exponential models for the rate of progression were computed resorting to the $\mathrm{R}$ programming language [23], whereas the linear regression models were computed using IBM SPSS v25 with an adopted statistical significance level of 0.05 .

\section{Results}

The number of confirmed infected cases of COVID-19 were initially analysed by plotting them against time. (Figure 2) depicts the curves obtained for the provinces that at the first time point (23td of January) had only one or two cases. The analysis of (Figure 2 ) shows that the number of accumulated cases is different between provinces and, in general, its rate decreases over time up to the point where it becomes null. Since the objective of the study was to analyse the rate of spread, we decided to use periods of 12 and 15 days to determine it. The initial 25 days were thought to be the most informative regarding the rate of spread. The fits of temporal evolution of confirmed cases of COVID-19 of the remaining provinces are not shown, but a similar profile can be obtained, leading to the same conclusion.

A two way ANOVA shows statistical differences $(F(1$, $450)=23.573 ; p<0.001$ ) between the size, 12 or 15 days of the period employed to compute the doubling time, but no statistical differences $(F(1,450)=0.047 ; p=0.828)$ between the number of samples taken from each province. This

Citation: Oliveiros $\mathrm{B}^{1,2,3^{*}}$, Caramelo $\mathrm{L}^{4}$, Ferreira $\mathrm{NC}^{1,3}$, Caramelo $\mathrm{F}^{1,2,3}$. Role of Temperature and Humidity in the Modulation of the Doubling Time of Covid-19 Cases Op Acc J Bio Sci \& Res 7(1)-2021.

DOI: 10.46718/JBGSR.2021.08.000199 


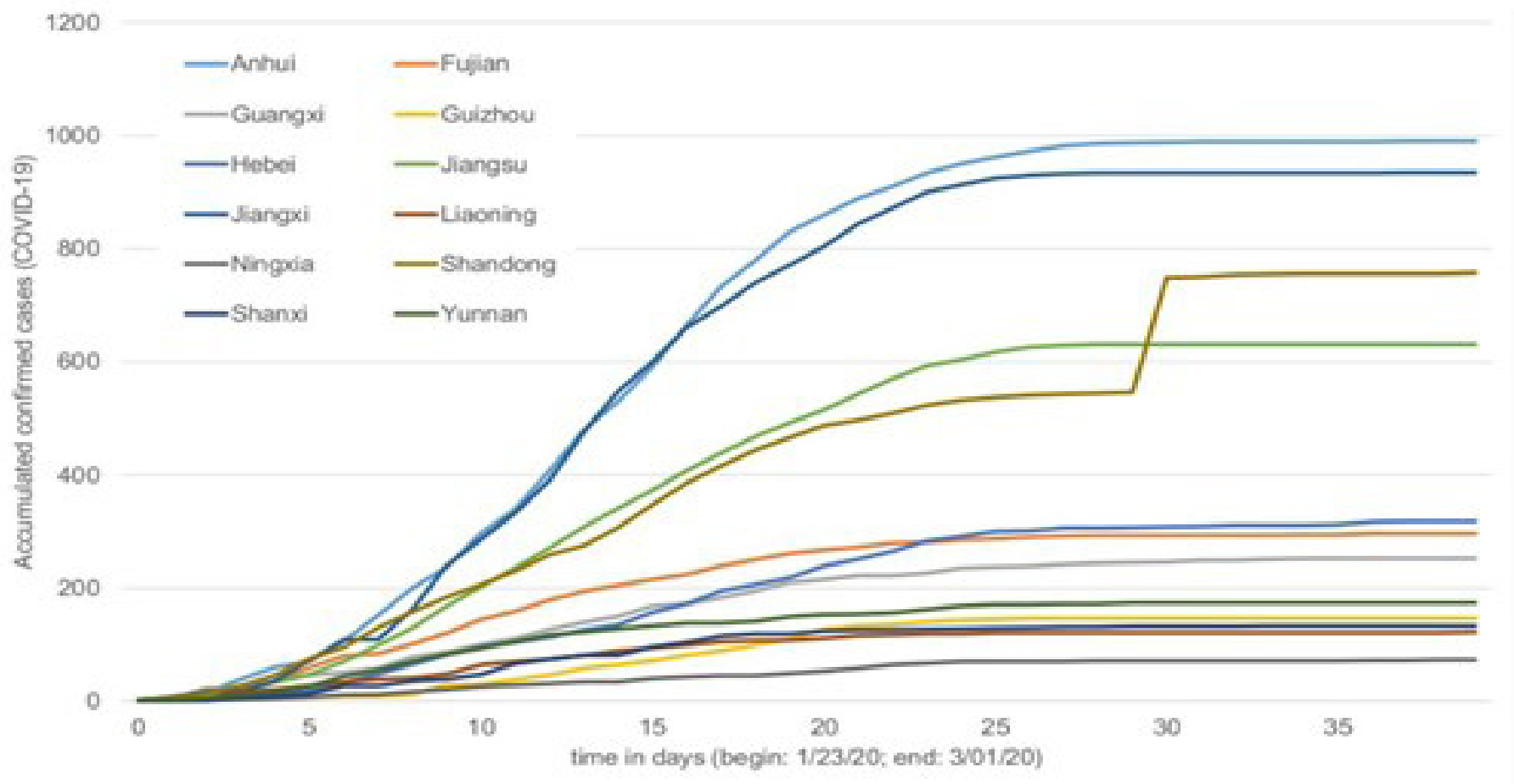

Figure 2: Accumulated confirmed cases of Covid-19 in function of time. Only the Provinces that had one or two cases at the beginning at the series are shown.

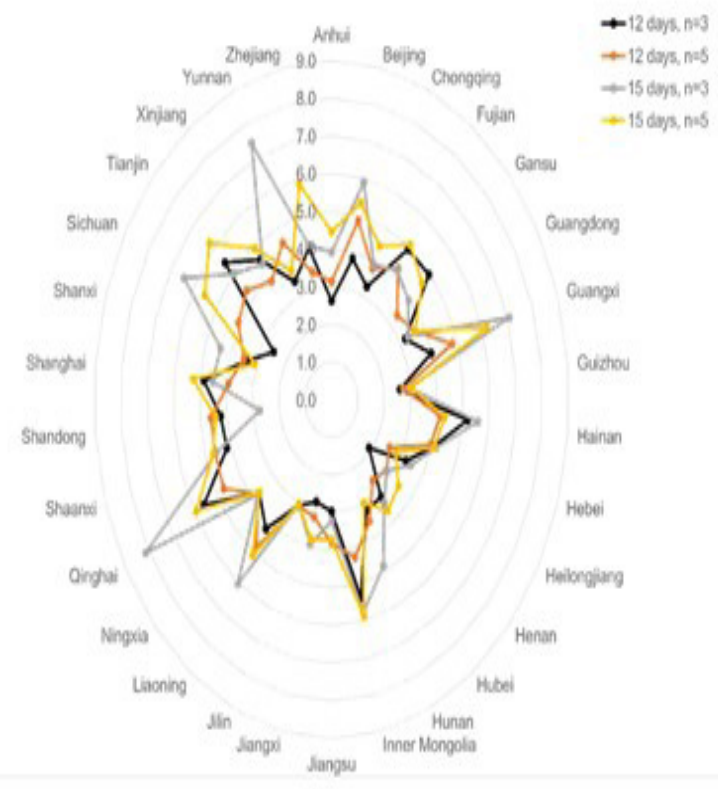

Figure 3: Doubling time for each province considering the different forms of calculation.

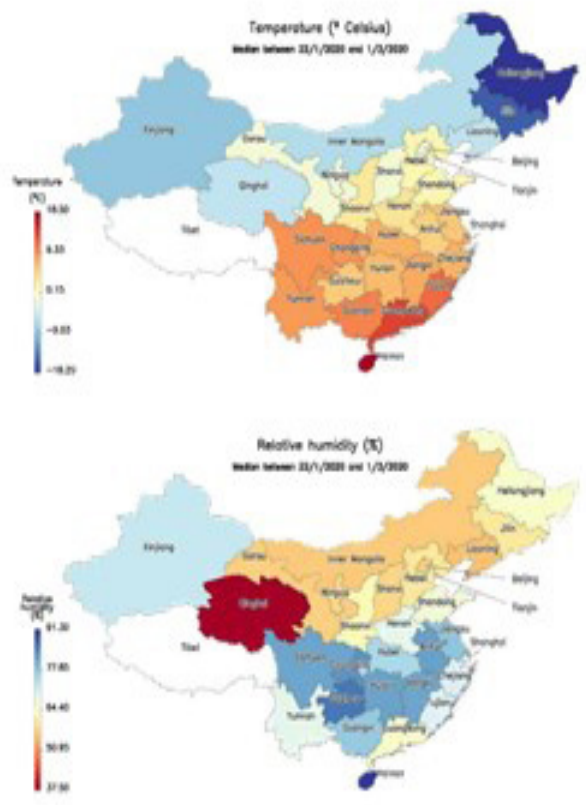

Figure 4: Median temperature (top) and relative humidity (bottom) of each province during the studied period. The corresponding values are shown in Annex I.

Citation: Oliveiros $\mathrm{B}^{1,2,3^{*}}$, Caramelo $\mathrm{L}^{4}$, Ferreira $\mathrm{NC}^{1,3}$, Caramelo $\mathrm{F}^{1,2,3}$. Role of Temperature and Humidity in the Modulation of the Doubling Time of Covid-19 Cases Op Acc J Bio Sci \& Res 7(1)-2021. 


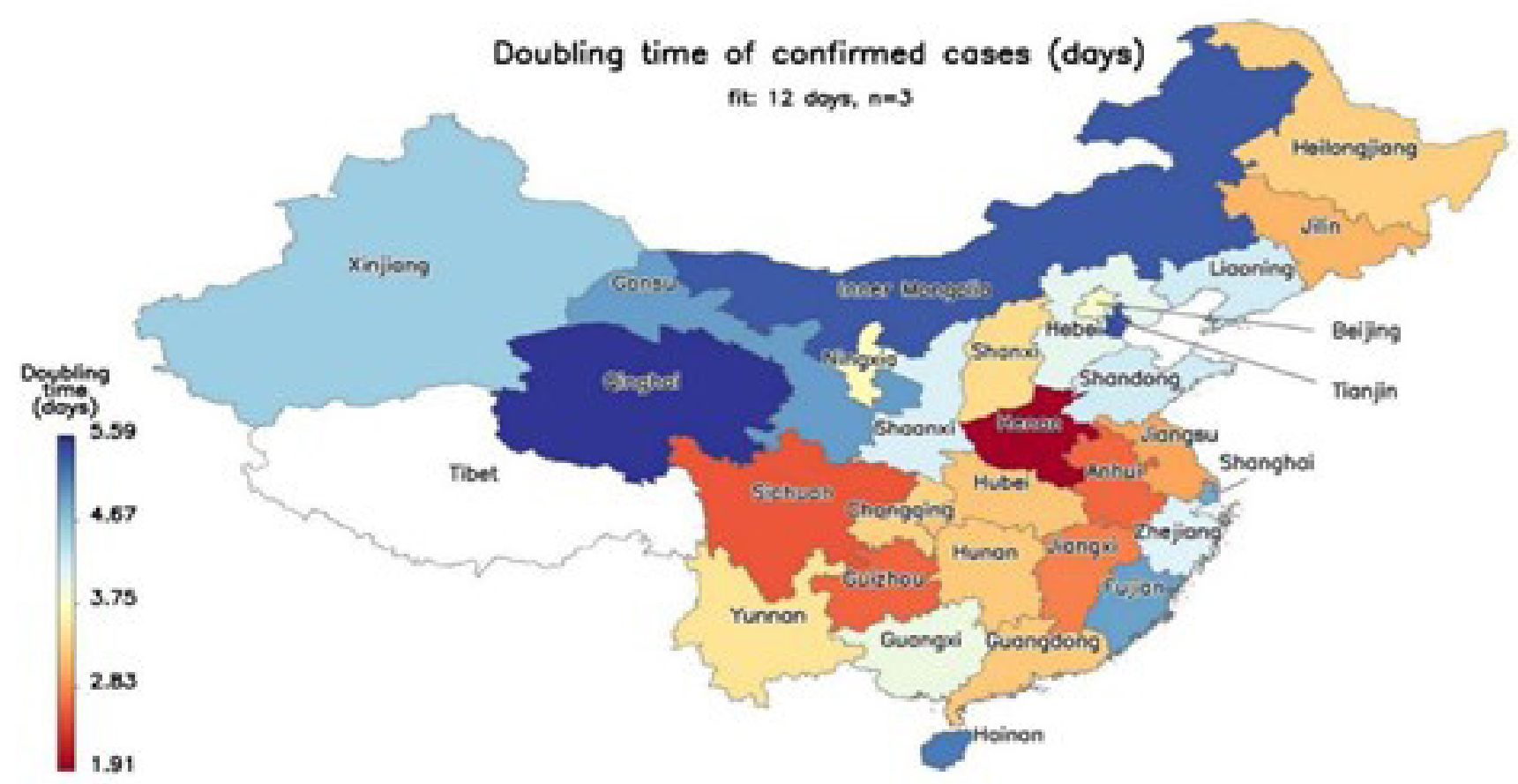

Figure 5: Doubling times obtained with one of the models (12 days, $n=3$ ).

Table 1: Statistics of the doubling time for two different time periods and two repetitions.

\begin{tabular}{|c|c|c|}
\hline & $\begin{array}{c}\text { Period of 12 } \\
\text { days }\end{array}$ & $\begin{array}{c}\text { Period of 15 } \\
\text { days }\end{array}$ \\
\hline $\begin{array}{c}\text { Number of } \\
\text { sampled }\end{array}$ & $3.78(1.34)$ & $4.53(1.68)$ \\
\hline periods = 3 & $1.64 / 7.05$ & $2.04 / 9.48$ \\
\hline $\begin{array}{c}\text { Number of } \\
\text { sampled }\end{array}$ & $3.82(1.27)$ & $4.42(1.53)$ \\
\hline periods = 5 & $1.67 / 7.05$ & $1.99 / 7.76$ \\
\hline
\end{tabular}

result is in agreement with the hypothesis that the doubling time changes with time. The average of the doubling time duplication was determined for each province (Figure 3).

Taking the values of doubling time and the meteorological variables, a linear regression was performed. (Table 2) shows, for the 4 conditions studied, the results achieved from the linear regressions, that assumed temperature and relative humidity as independent variables. Precipitation and wind speed did not reach statistical significance in any model (data not shown), thus (Table 2) only refers results for temperature and relative humidity.

The results obtained for all models are statistically meaningful and despite their variation, it is possible to perceive that the coefficients of regression (B) are not statistically different as their confidence intervals overlap. On the other hand, the amount of variation explained, given by the adjusted $\mathrm{R}$ square value, differs between models. The model based on 12 days and 3 sampled periods is able to explain $18 \%$ of the variance in the doubling time, which means that temperature and relative humidity alone may describe $18 \%$ of the variation of confirmed COVID-19 infections. More importantly, the signal and value of the coefficients of regressors are of utterly importance to understand how the spread of COVID-19 is expected to be affected by temperature and relative humidity. According to all models, temperature increases the doubling time, which means that it delays the spread of COVID-19. Relative humidity, on the contrary, benefits it. The models differ, however, on the amount of contribution: for example, in the best scenario (model: 15 days, $n=3$ ) the doubling time is increased by 0.090 days for each Celsius degree increase, and is increased by 0.072 days for each unit decrease of the relative humidity value (Figures $4 \& 5$ ).

\section{Discussion and Conclusion}

In this work, the way temperature and relative humidity affect the doubling time of COVID-19 spreading was determined. Results suggest that temperature correlates positively with the doubling time and negatively with relative humidity. This means that, with spring and summer,

Citation: Oliveiros $\mathrm{B}^{1,2,3^{*}}$, Caramelo $\mathrm{L}^{4}$, Ferreira $\mathrm{NC}^{1,3}$, Caramelo $\mathrm{F}^{1,2,3}$. Role of Temperature and Humidity in the Modulation of the Doubling Time of Covid-19 Cases Op Acc J Bio Sci \& Res 7(1)-2021.

DOI: 10.46718/JBGSR.2021.08.000199 
Table 2: Results of the linear regressions between doubling time, temperature and relative

\begin{tabular}{|c|c|c|c|}
\hline Condition & Model significance & & Coeficents of regression (B) \\
\hline $\begin{array}{c}12 \text { days } \\
n=3\end{array}$ & $\begin{array}{c}\mathrm{F}(2,81)=10.109 ; \\
\mathrm{p}<0.001\end{array}$ & 0.180 & $\begin{array}{c}\text { T: } B=+0.048 \text { CI95\%[0.005; 0.092]; } p=0.028 \\
H: B=-0.066 \text { CI95\%[-0.095; }-0.036] ; p<0.001\end{array}$ \\
\hline $\begin{array}{c}12 \text { days } \\
n=5\end{array}$ & $\begin{array}{c}F(2,140)=8.702 \\
p<0.001\end{array}$ & 0.098 & $\begin{array}{c}\text { T: } B=+0.049 \text { CI95\%[0.018; 0.081]; } p=0.002 \\
\text { H: } B=-0.050 \text { CI95\%[-0.074; }-0.026] ; p<0.001\end{array}$ \\
\hline $\begin{array}{c}15 \text { days } \\
n=3\end{array}$ & $\begin{array}{c}F(2,83)=6.792 \\
p=0.002\end{array}$ & 0.120 & $\begin{array}{c}\text { T: } B=+0.090 \text { CI95\%[0.033; 0.148]; } p=0.002 \\
\text { H: } B=-0.072 \text { CI95\%[-0.112; }-0.031] ; p=0.001\end{array}$ \\
\hline $\begin{array}{c}15 \text { days } \\
n=5\end{array}$ & $\begin{array}{c}F(2,138)=6.246 \\
p=0.003\end{array}$ & 0.070 & $\begin{array}{c}\text { T: } B=+0.061 \text { CI95\%[0.020; 0.103]; } p=0.004 \\
\text { H: } B=-0.051 \text { CI95\%[-0.080; }-0.021] ; p=0.001\end{array}$ \\
\hline
\end{tabular}

the rate of progression of COVID-19 is expected to be slower. Still, these two variables contribute at maximum to $18 \%$ of the variation, being the remaining $82 \%$ related to other factors such as containment measures, general health policies, population density, transportation, cultural aspects, etc. Besides, the direct impact is also small: for example, if temperature raises $20^{\circ} \mathrm{C}$, it is expected that in the best-case scenario the doubling time increases on average

1.8 days. These results are in agreement with other studies that suggested that the aerosol spread of the influenza virus is both dependent upon relative humidity and temperature, although performed in animal models [14], and that the virus survival in droplets is higher at high relative humidity levels with a significant

decrease on its infectivity rate at mid-levels of relative humidity [15].

Wei Lo et al 17 recently reported a statistically significant association between absolute relative humidity and mean temperature on COVID-19 spread among China provinces. Furthermore, they have concluded that transmission and exponential growth of confirmed cases are occurring in China provinces in relative humidity conditions ranging from cold and dry (Jilin or Heilongjiang) to tropical (Guangxi or Singapore), suggesting that changes in weather, as expected by the arrival of spring and summer, will not necessarily lead to declines in outbreak unless extensive public health interventions are implemented, and that further studies on the effects of meteorology on COVID-19 transmission are needed.
On the other hand, Jin Bu et al 18 reached the conclusion that continuous warm and dry weather is conducive to the survival of the 2019-nCoV and speculate that conditions such as temperature ranging from 13 to $19^{\circ} \mathrm{C}$ and relative humidity between $50 \%$ and $80 \%$ are suitable for the survival and transmission of this new coronavirus. However, their predictions were performed using SARS data and meteorological conditions at that time and, as they report, 2019-nCoV has a high basic case reproduction number (RO) lying between 2.2 to 6.7 , causing much more infections than SARS.

Moreover, Mao Wang et al 19 have recently submitted a paper supporting that temperature could change the COVID-19 transmission and that there might be an optimal temperature for the viral transmission, suggesting that colder regions in the world should adopt strictest control measures. Yuwen Cai [20] did not find any correlation between the growth rate of the epidemy and daily mean temperature in either Wuhan or Hunan but found a weak correlation between the mortality in confirmed cases and daily mean temperature both in Wuhan $(r=-0.441)$ and Hubei ( $r=-0.440)$, although not adjusted for the useof three makeshift hospitals, which proved to be effective. The main focus of this work was to assess the relationship between the rate of spread of COVID-19 and some meteorological variables, which determines the type of model adopted.

Although the reproduction number, 0 , is the parameter widely accepted to characterize the velocity of spreading, there are different forms of computing it, which tend to lead

Citation: Oliveiros $\mathrm{B}^{1,2,3^{*}}$, Caramelo $\mathrm{L}^{4}$, Ferreira $\mathrm{NC}^{1,3}$, Caramelo $\mathrm{F}^{1,2,3}$. Role of Temperature and Humidity in the Modulation of the Doubling Time of Covid-19 Cases Op Acc J Bio Sci \& Res 7(1)-2021.

DOI: 10.46718/JBGSR.2021.08.000199 
to different results [24]. On the other hand, the 0 calculation is generally based on assumptions about the epidemic phenomenon such as serial interval distribution [25] or "the population is closed, that all cases are observed, and use daily case counts only" [26]. For the reasons mentioned, we opted for a simple/naive model that could assimilate the principal aspects of the variation of COVID-19 cases and translate it into a straightforward measurement that could be easily comprehended. Obviously, this model has several drawbacks, mostly regarding the optimal period where an exponential growth is verified. We studied two different periods sizes and a random starting point aiming to analyse the impact of this aspect and as an attempt to mitigate its consequences. The doubling time values vary with the period size $26 \%$ at maximum (Table 1 ), which is unneglectable. Even so, this difference only affects slightly the regression coefficients of temperature and relative humidity, since they do not show statistical differences. The average incubation time accepted for the COVID-19 is 3 days, but it can be as long as 14 days. This is a problem, since when the results of the tests are provided, the transmission has already occurred and the meteorological variables should be given for that period. Therefore, the use of a random starting point and two periods to compute the duplication time are a form to compensate the uncertainty caused by the incubation time. This effect is particularly important for the first points in each period, which is balanced by the use of different starting points, and a time period of 15 days that can accommodate the great majority of transmission. On the other hand, as we are looking at the first days of the epidemic with exponential growth, the later points have more weight than the initial ones. Moreover, we use the median of temperature and relative humidity, instead of the average, of this random sliding time window because it is a robust measure against outliers and translates $50 \%$ of the results. Besides, the maximum interquartile range that we found was $4.4{ }^{\circ} \mathrm{C}$ for temperature and $29.1 \%$ for relative humidity (Annex I).

Another point of possible bias is the COVID-19 data that do not cover all provinces from the beginning of the outbreak, making it difficult to homogeneously study in all provinces the time period that corresponds to the exponential growth. A different concern is the possibility that containment measures may affect the results, and that was the reason why we selected China. To obtain meteorological variety we needed a vast region that could be the concatenation of several countries or just one large country. China seems to be the most appropriate since it has a vast territory that is governed under the same main rules and that adopted similar containment measures and testing policies in a short period of time. Regional differences certainly exist and this could be one of the factors that the linear models did not capture, as we mentioned before. Nonetheless, it is accepted that the results of containment measures have a delay of at least 2 weeks, and since we are analyzing the first days, these effects would not interfere with our analysis.

The meteorological variables in this study were obtained for locations near the center of provinces, which typically do not correspond to the average location of the population. Measurements that better represent the central tendency of the meteorological variables felt by the general population of a particular region are currently being implemented. A final remark about the short average doubling time values obtained (3.78 to 4.53 days - Table 1 ), which should be a motive of concern. For each doubling time, the number of infected doubles, so one month of sustained growth at a conservative pace of 5 days means an increase of the number of infected by a factor of 64 .

\section{Contributor Ship Statement}

All the authors have equally contributed in the implementation of the computational model, assessment of the results and writing of the manuscript.

\section{Competing Interests}

There are no competing interests.

Funding

Funded by National Funds via FCT (Foundation for Science and Technology) through the Strategic Project UIDB/04539/2020 and UIDP/04539/2020 (CIBB).

\section{Data Sharing Statement}

Data used in this work are publicly available and cited accordingly.

\section{References}

1. World Health Organization (2020) Novel coronavirus - China. Geneva, Switzerland: World Health Organization.

2. Wang C, Hornby PW, Hayden FG, Gao GF (2020) A novel coronavirus outbreak of global health concern. Lancet 395(10223): 470-473.

3. Hui DS, Azhar EI, Madani TA, Ntoumi F, Kock R, Dar O, et al. (2020) The continuing 2019-nCoV epidemic threat of novel coronaviruses to global health - the latest 2019 novel coronavirus, outbreak in Wuhan, China. Int J Infect Dis 91: 264.

4. Zhu N, Zhang D, Wang W, Li XW, Yang B, Song JD, et al. (2020) A novel coronavirus from patientswith pneumonia in China, 2019. N Engl J Med. N Engl J Med 382: 727-733.

5. Chan JFW, Kok KH, Zhu Z, Chu H, To KKW, Yuan SF, et al. (2020) Genomic characterization of the 2019 novel human-pathogenic coronavirus isolated from a patient with atypical pneumonia after visiting Wuhan. Emerg Microbs Infect 9(1): 221-236.

6. Tan WJ, Zhao X, Ma XJ, Wang WL, Niu PH, Xu WB, et al. (2020) A novel coronavirus genome identified in cluster of pneumonia cases-Wuhan, China 2019-2020. China CDC Weekly 2(4): 61- 62.

7. Paules CI, Marston HD, Fauci AS (2020) Coronavirus infection-more

Citation: Oliveiros $\mathrm{B}^{1,2,3^{*}}$, Caramelo $\mathrm{L}^{4}$, Ferreira $\mathrm{NC}^{1,3}$, Caramelo $\mathrm{F}^{1,2,3}$. Role of Temperature and Humidity in the Modulation of the Doubling Time of Covid-19 Cases Op Acc J Bio Sci \& Res 7(1)-2021.

DOI: 10.46718/JBGSR.2021.08.000199 
than just the common cold. JAMA.

8. Munster VJ, Koopmans M, van Doremalen N, van Riel D, de Wit E (2020) A novel coronavirus emerging in China - key questions for impact assessment. N Engl J Med 382: 692-694.

9. Huang C, Wang Y, Li X, Ren L, Zhao J, Hu Y, et al. (2020) Clinical features of patients infected with 2019 novel coronavirus in Wuhan, China. Lancet. 395(10223): 497-506.

10. Chan JFW, Yuan S, Kok KH, To KKW, Chu H, Yang J, et al. (2020) A familial cluster of pneumonia associated with the 2019 novel coronavirus indicating person-to-person transmission: a study of a family cluster. Lancet 395: 497-506.

11. The Nove Coronavirus Pneumonia Emergency Response Epidemiology Team. The Epidemiological Characteristics of an Outbreak of 2019 Novel Coronavirus Diseases (COVID-19). China CDC Weekly 2020; 41(2): 145-151.

12. Caramelo F, Ferreira N, Oliveiros B (2020) Estimation of risk factors for COVID-19 mortality - preliminary results. Preprint at Med RXIV.

13. https://gisanddata.maps.arcgis.com/apps/opsdashboard/index.html\#/ bda7594740fd402

14. Lowen AC, Mubareka S, Steel J, Palese P (2007) Influenza Virus Transmission is Dependent on Relative Humidity and Temperature. PLoS Pathol 3(10): e151.

15. Prussin AJ, Schwake DO, Lin K, Gallagher DL, Buttling L, et al. (2018) Survival of the Enveloped Virus Phi6 in Droplets as a Function of Relative Humidity, Absolute Humidity, and Temperature. Appl Environ Microbio 84(12): e00551-18.

16. Simmering JE, Polgreen LA, Hornick DB, Sewell DK, Polgreen PM (2017)
Weather-Dependent Risk for Legionnaires' Disease, United States. Emerg Infect Dis 23(11): 1843-1851.

17. Luo W, Majumder MS, Liu D, Poirier C, Mandl KD, et al. (2020) The role of absolute humidity on transmission rates of the COVID-19 outbreak. Preprint at Med RXIV.

18. Bu J, Peng DD, Xiao H, Yue Q, Han Y, et al. (2020) Analysis of meteorological conditions and prediction of epidemic trend of 2019nCoV infection in 2020. Preprint at Med RXIV.

19. Wang M, Jiang A, Gong L, Luo L, Guo W, et al. (2020) Temperature significant change COVID-19 Transmission in 429 cities. Preprint at Med RXIV.

20. Cai Y, Huang Sr T, Liu Sr X, Xu Sr G (2020) The Effects of "Fangcang, Huoshenshan, and Leishenshan" Makeshift Hospitals and Temperature on the Mortality of COVID-19. Preprint at Med RXIV.

21. https://api.meteostat.net/

22. https://github.com/CSSEGISandData/COVID- 19/tree/master/csse_ covid_19_data/csse_covid_19_time_series

23. R Core Team (2017) R: A language and environment for statistical computing. R Foundation for Statistical Computing, Vienna, Austria.

24. Obadia T, Haneef R, Boëlle P (2012) The R0 package: a toolbox to estimate reproduction numbers for epidemic outbreaks. BMC Med Inform Decis Mak 12: 147.

25. Hens N, Van Ranst M, Aerts M, Robesyn E, Van Damme P, et al. (2011) Estimating the effective reproduction number for pandemic influenza from notification data made publicly available in real time: a multicountry analysis for influenza A/H1N1v 2009 29(5): 896-904.

26. White LF, Pagano M (2008) Transmissibility of the influenza virus in the 1918 pandemic. PLoS One 3(1): e1498.

*Corresponding author: Bárbara Oliveiros, Email: boliveiros@fmed.uc.pt

Next Submission with BGSR follows:

- Rapid Peer Review

- Reprints for Original Copy

- E-Prints Availability

- Below URL for auxiliary Submission Link: https://biogenericpublishers.com/submit-manuscript/ 